\title{
Assaying Biomarkers via Real-Time Measurements of the Effective Relaxation Time of Biofunctionalized Magnetic Nanoparticles Associated with Biotargets
}

\author{
Shu-Hsien Liao, ${ }^{1}$ Jean-Hong Chen, ${ }^{2}$ Yu-Kai Su, ${ }^{1}$ Kuen-Lin Chen, \\ Herng-Er Horng, ${ }^{1}$ and Hong-Chang Yang ${ }^{4}$ \\ ${ }^{1}$ Institute of Electro-Optical Science and Technology, National Taiwan Normal University, Taipei 116, Taiwan \\ ${ }^{2}$ Department of Materials Engineering, Kun Shan University, Tainan 710, Taiwan \\ ${ }^{3}$ Department of Physics, National Chung Hsing University, Taichung 402, Taiwan \\ ${ }^{4}$ Department of Electro-Optical Engineering, Kun Shan University, Tainan 710, Taiwan \\ Correspondence should be addressed to Shu-Hsien Liao; shliao@ntnu.edu.tw
}

Received 12 September 2014; Accepted 9 February 2015

Academic Editor: Yali Cui

Copyright (C) 2015 Shu-Hsien Liao et al. This is an open access article distributed under the Creative Commons Attribution License, which permits unrestricted use, distribution, and reproduction in any medium, provided the original work is properly cited.

\begin{abstract}
An assay of biomarkers consisting of alpha-fetoprotein (AFP) is reported. Real-time measurements of the effective relaxation time $\tau_{\text {eff, }}$, when the biofunctionalized magnetic nanoparticles (BMNs) were conjugating with biotargets, were made. The BMNs are antialpha-fetoprotein (antiAFP) coated onto dextran-coated iron oxide nanoparticles labeled as $\mathrm{Fe}_{3} \mathrm{O}_{4}$-antiAFP. It was found that the effective relaxation time, $\tau_{\text {eff }}$, increases as the association of AFP and $\mathrm{Fe}_{3} \mathrm{O}_{4}$-antiAFP evolves. We attribute this to the enhanced Brownian motion of BMNs when magnetic clusters are present during the conjugation. We found that saturation magnetization, $M_{s}$, increases when the concentration of AFP increases. This is due to the fact that more magnetic clusters are associated in the reagent, and therefore the $M_{s}$ increases when the concentration of AFP increases. The change of effective relaxation time and saturation magnetization shows a behavior of logistic function, which provides a foundation for assaying an unknown amount of biomolecules. Thus, we demonstrate sensitive platforms for detecting AFP by characterizing $\tau_{\text {eff }}$. The detection platform is robust and easy to use and shows promise for further use in assaying a broad number of biomarkers.
\end{abstract}

\section{Introduction}

Assaying biomarkers based on electrical [1], optical [2,3], and magnetic technologies $[4,5]$, and so forth, has been reported, in which, immuomagnetic assays based on magnetic detection using biofunctionalized magnetic nanoparticles (BMNs) have received considerable attention. Magnetic detection has a negligible magnetic background, and thus high detection sensitivity and specificity can be achieved. Magnetic detection can be done through the measurements of magnetic relaxation [6, 7], magnetic remanence [8], immunomagnetic alternating current (ac) susceptibility reduction [9], saturated magnetization [10], spin-spin relaxation [11], and so forth. Detection sensors include SQUIDs [12], magnetoresistive sensors [13], Hall sensors [14], and on-chip magnetic resonance sensors [15]. Many of these techniques, however, have a time-consuming stage of sample preparation. On the contrary, detecting biomarkers using the immunomagnetic reduction (IMR) assay [9], which detects the change of the amplitude at the mixed-frequency. The method shows high sensitivity and specificity. Additionally, sample preparation is simple and easy to operate. Some studies involving this approach include the detecting of cytokines [16] and alphafetoprotein (AFP) for human expressing tumors in clinical research [17].

In contrast to direct detection of magnetic susceptibility reduction at the mixed-frequency, we have recently developed a new magnetic sensing platform, single-frequency ac susceptometer for biodetection [18]. The ac susceptometer detects the phase lag of the magnetization, $\mathbf{M}(t)$, of BMNs 
with respect to the applied field, $\mathbf{H}(t)$, as the detection mechanism. During the association of BMNs with biomarkers the magnetic clusters are conjugated, which changes the phase lag and in turn changes the time-dependent relaxation rate. In this work, the detection platform was used to assay the biomolecule abundance of alpha-fetoprotein (AFP) by measuring the time-dependent effective relaxation time and saturation magnetization of reagents conjugating with the AFP. Reagents consisted of antiAFP coated onto the $\mathrm{Fe}_{3} \mathrm{O}_{4}$ magnetic oxides and labeled as $\mathrm{Fe}_{3} \mathrm{O}_{4}$-antiAFP. AFP can be used as a biomarker to detect liver tumors. A level above $500 \mathrm{ng} / \mathrm{mL}$ of AFP in adults can be indicative of hepatocellular carcinoma, germ cell tumors, and metastatic cancers of the liver. Biomarkers of AFP are first characterized by measuring the phase lag $\theta(t)$, which is between the applied magnetic field $\mathbf{H}(t)$ and magnetization $\mathbf{M}(t)$. Then the dynamic effective relaxation time $\tau_{\text {eff }}$ was estimated through the following formula: $\tan \theta=\omega \tau_{\text {eff }}$, where $\omega$ is the exciting angular frequency. It was found that the change of effective relaxation time, $\Delta \tau_{\text {eff }}$, increases when the AFP completed the association with $\mathrm{Fe}_{3} \mathrm{O}_{4}$-antiAFP. Additionally, the saturation magnetization increases as the concentration of AFP increases. We attribute this to the presence of magnetic clusters during the conjugation. A detection sensitivity better than $100 \mathrm{ng} / \mathrm{mL}$ of AFP is demonstrated. The detection platform is robust and easy to use, showing promise for further use in a broad number of biomedical applications, such as viruses, proteins, and tumor markers.

The reagent for assaying AFP consists of magnetic nanoparticles (MF-DEX-0060, MagQu, New Taipei, Taiwan) functionalized with antibodies (ab40942; Abcam, Cambridge, MA), against AFP (EA502-Q1053; EastCoast Bio, North Berwick, ME). These magnetic nanoparticles are dispersed in phosphoryl buffer solution with $\mathrm{pH}=7.4$. Measured with a vibration sample magnetometer, the concentration of magnetic reagents containing $\mathrm{Fe}_{3} \mathrm{O}_{4}$-antiAFP was $0.3 \mathrm{emu} / \mathrm{g}$ which corresponds to a concentration of $1.2 \mathrm{mg}-\mathrm{Fe} / \mathrm{mL}$.

\section{Experiments}

2.1. Biodetection System. Figure 1 shows the unique design of the sensitive ac magnetic susceptometer. The sensing coils consist of an input coil, pick-up coil, and compensation coil. The input coil, pick-up coil, and compensation coil are, respectively, 4800 turns (4.9 $\Omega$ ), 1260 turns (1.2 $\Omega$ ), and 2 turns of wound copper coils. The pick-up coil consisted of two coils wound in opposite directions. The excitation frequency was $9 \mathrm{kHz}$. The signal from the function generator is first attenuated with a variable resistor $(\sim 1 \mathrm{M} \Omega)$. A compensation coil was applied to improve the balance of the detection coil. A balance of 30 parts per trillion (ppt) is achieved in such a sensing unit. Due to the nonlinear magnetic characteristics of magnetic nanoparticles, when the excitation frequency $f_{1}(t)$ is applied to the input coil, an excited signal with $n f_{1}(t)$ components will be generated in the pick-up coil. In the present study, the dynamic $\theta(t)$ and amplitude, $\chi_{\mathrm{amp}}$, of the fundamental component, $f_{1}(t)$, are detected through lock-in detection.

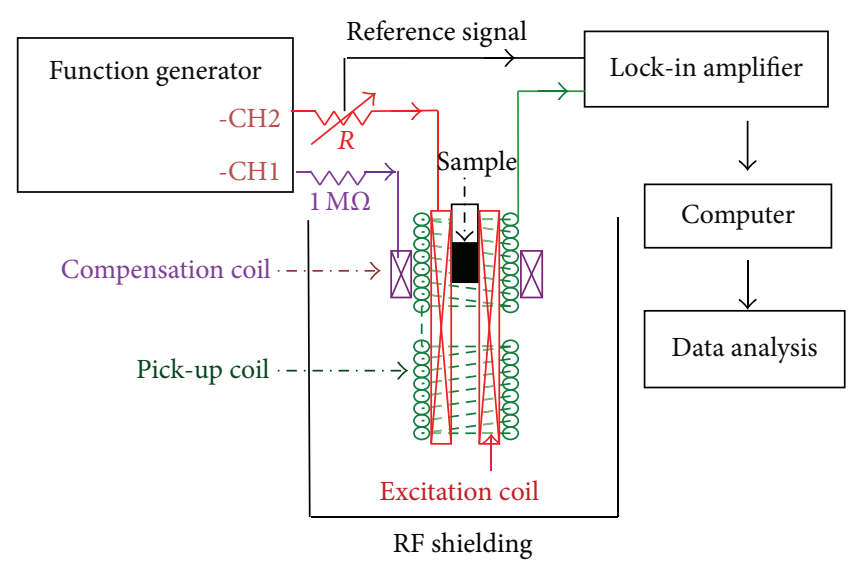

FIGURE 1: The detection scheme of an ac magnetic susceptometer with a lock-in detection technique.

2.2. Detection Mechanism. Due to the molecular interaction, BMNs are associated with biomarkers and the magnetic clusters are conjugated. The magnetization from magnetic clusters will affect the physical properties of the BMNs during the association of BMNs with the biomarkers, for instance, the dynamic phase lag $\theta(t)$ of $\mathbf{M}(t)$ with respect to $\mathbf{H}(t)$ in ac susceptibility and the saturation magnetization, $M_{s}$, and so forth. By characterizing the dynamic $\theta(t)$ or $M_{s}$, we can therefore determine the unknown amount of biomarkers.

We consider the ac magnetic susceptibility of BMNs, $\chi(\omega)$, in an applied ac magnetic field. $\chi(\omega)$ is a function of $\tau_{\text {eff }}$ and exciting angular frequency $\omega$. The $\chi(\omega)$ can be expressed as follows [19]:

$$
\begin{aligned}
\chi_{\mathrm{ac}} & =\chi^{\prime}+i \chi^{\prime \prime} \\
& =\chi_{0}\left\{\left\{\frac{1}{\left[1+\left(\omega \tau_{\mathrm{eff}}\right)^{2}\right]}\right\}^{2}+\left\{\frac{\left(\omega \tau_{\mathrm{eff}}\right)}{\left[1+\left(\omega \tau_{\mathrm{eff}}\right)^{2}\right]}\right\}^{2}\right\}^{1 / 2} e^{-i \theta},
\end{aligned}
$$

where $\chi_{0}$ is the component of $\chi_{\mathrm{ac}}$ at $\omega=0, i=(-1)^{1 / 2}$, $\chi^{\prime}=\chi_{0}\left\{1 /\left[1+\left(\omega \tau_{\text {eff }}\right)^{2}\right]\right\}, \chi^{\prime \prime}=\chi_{0}\left\{\left(\omega \tau_{\text {eff }}\right) /\left[1+\left(\omega \tau_{\text {eff }}\right)^{2}\right]\right\}$, and $\chi^{\prime \prime} / \chi^{\prime}=\tan \theta . \theta$ is the phase lag of the time-varying magnetization $\mathbf{M}$ with respect to the applied ac magnetic field $\mathbf{H}$, and $\tau_{\text {eff }}$ is the effective relaxation rate. Using (1) and (2), we obtained $\tan \theta=\omega \tau_{\text {eff }}$ with $\chi^{\prime \prime} / \chi^{\prime}=\tan \theta$. The $\tau_{\text {eff }}$ of BMNs can be written as

$$
\frac{1}{\tau_{\mathrm{eff}}}=\frac{1}{\tau_{B, \mathrm{eff}}}+\frac{1}{\tau_{N, \mathrm{eff}}},
$$

where $1 / \tau_{B \text {,eff }}$ is the effective relaxation rate due to Brownian relaxation and $1 / \tau_{N \text {,eff }}$ is the effective relaxation rate due to Brownian relaxation due to Néel relaxation. We note that $\tau_{\text {eff }}$ characterizes the ability to retain the magnetization after the applied dc field is removed and it reflects the influences of clustered magnetic nanoparticles on Brownian and Néel relaxation.

Since $\theta$ is related to the effective relaxation time, $\tau_{\text {eff, }}$, by the relation $\tan \theta=\omega \tau_{\text {eff }}$, where $\omega$ is the excitation 


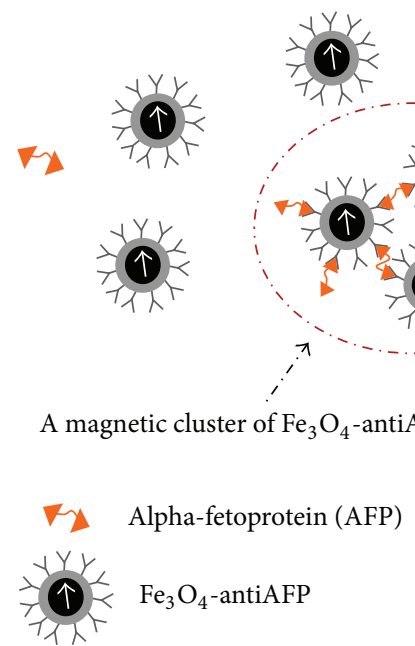

(a)

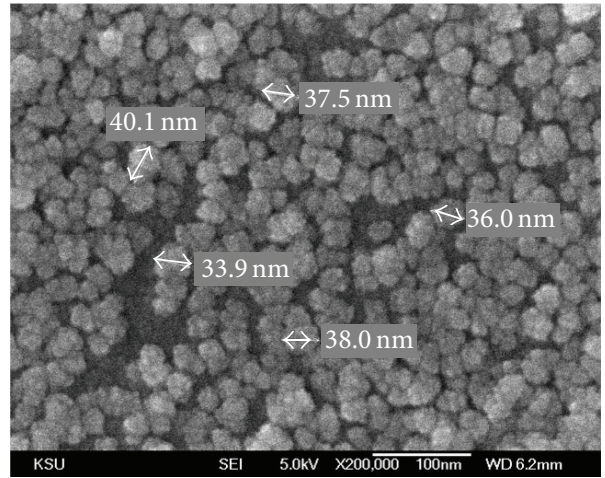

(b)

FIgURE 2: A cartoon drawing of $\mathrm{AFP}, \mathrm{Fe}_{3} \mathrm{O}_{4}$-antiAFP, and magnetic clusters of $\mathrm{Fe}_{3} \mathrm{O}_{4}$-antiAFP-AFP.

frequency, we can understand the dynamic characteristics of $\tau_{\text {eff }}(t)$ by measuring the time-dependency of $\theta(t)$ with a lockin amplifier. Furthermore, the formation of the magnetic clusters during conjugation affects the relaxation and therefore changes the $\tau_{\text {eff }}(t)$. To quantitatively describe this assay we define $\Delta \tau_{\text {eff }} / \tau_{0}=\left[\tau_{\text {eff }}(t=0)-\tau_{\text {eff }}(t=\infty)\right] / \tau_{\text {eff }}(t=0)$, where $\Delta \tau_{\text {eff }}=\left[\tau_{\text {eff }}(t=0)-\tau_{\text {eff }}(t=\infty)\right]$ and $\tau_{\text {eff }}(t=0)=\tau_{0}$. We will analyze $\Delta \tau_{\text {eff }} / \tau_{0}$ to determine amount of biomarkers.

2.3. Reagents. The reagent for assaying AFP consists of magnetic nanoparticles (MF-DEX-0060, MagQu, New Taipei, Taiwan) functionalized with antibodies (ab40942; Abcam, Cambridge, MA) against AFP (EA502-Q1053; EastCoast Bio, North Berwick, ME). These magnetic nanoparticles are dispersed in phosphoryl buffer solution (PBS). The BMNs for assaying AFP are $\mathrm{Fe}_{3} \mathrm{O}_{4}$-antiAFP dispersed in phosphate buffered saline solution with a $\mathrm{pH}$ value of 7.4. The magnetic core is $\mathrm{Fe}_{3} \mathrm{O}_{4}$, which is coated with dextran. Cartoons of the AFP, $\mathrm{Fe}_{3} \mathrm{O}_{4}$-antiAFP, and conjugated magnetic clusters of $\mathrm{Fe}_{3} \mathrm{O}_{4}$-antiAFP-AFP are represented in Figure 2(a) while the SEM picture of the reagent $\mathrm{Fe}_{3} \mathrm{O}_{4}$-antiAFP is shown in Figure 2(b). The size of the $\mathrm{Fe}_{3} \mathrm{O}_{4}$-antiAFP is $\sim 37 \mathrm{~nm}$, which is close to the average hydrodynamic diameter of $\sim 45 \mathrm{~nm}$ detected with the dynamic light scattering. To achieve the association between AFP and $\mathrm{Fe}_{3} \mathrm{O}_{4}$-antiAFP, we used the saturated magnetization of a $0.3 \mathrm{emu} / \mathrm{g}$ magnetic reagent. To assay AFP, $60 \mu \mathrm{L}$ magnetic reagents consisting of $\mathrm{Fe}_{3} \mathrm{O}_{4}{ }^{-}$ antiAFP are mixed with $60-\mu \mathrm{L}$ AFP of different concentrations, varied from $10 \mathrm{ng} / \mathrm{mL}$ to $10000 \mathrm{ng} / \mathrm{mL}$.

\section{Results and Discussion}

3.1. Time Dependence of Phase Lag $\theta$. Figure 3 shows the time dependence of $\theta(t)$ after mixing the reagents with AFP at $300 \mathrm{~K}$. It was found that $\theta(t)$ is independent of time before the reagent is mixed with the to-be-detected AFP. After mixing the reagent with AFP, the $\theta(t)$ increases

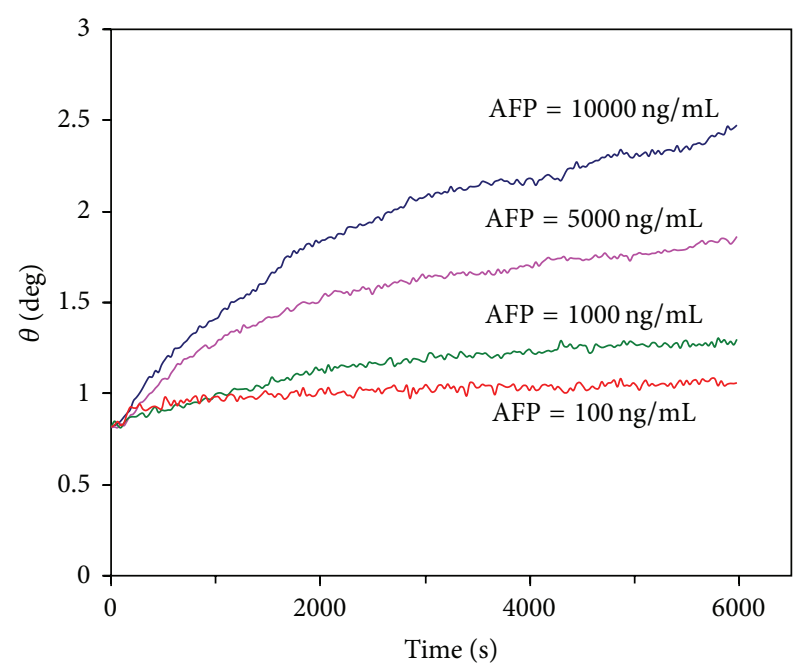

FIGURE 3: Time-dependent $\theta$ for assaying $10000 \mathrm{ng} / \mathrm{mL}, 5000 \mathrm{ng} /$ $\mathrm{mL}, 1000 \mathrm{ng} / \mathrm{mL}$, and $100 \mathrm{ng} / \mathrm{mL}$ of AFP.

monotonically and reaches saturated behavior when the association is completed. The $\theta(t=0)=0.8$ degrees was observed at $t=0$ and $\theta(t=6000 \mathrm{~s})$ increases monotonically to 1.3 degrees at $t=6000 \mathrm{~s}$ when we assay $1000 \mathrm{ng} / \mathrm{mL}$ of AFP, and a change of the phase lag $\Delta \theta=0.5$ degrees was detected. The $\theta(t=0)=0.8$ degrees at $t=0$ and $\theta(t=6000 \mathrm{~s})$ increases to 1.05 degrees at $t=6000 \mathrm{~s}$ when we assay $100 \mathrm{ng} / \mathrm{mL}$ of AFP, and a change of the phase lag $\Delta \theta=0.25$ degrees was observed.

Figure 4 shows $\Delta \theta / \theta_{0}(t=0)$ as a function of AFP concentration in a semilog plot, where $\Delta \theta=\theta(t=6000)-$ $\theta(t=0)$. The $\Delta \theta / \theta_{0}=1.76(\Delta \theta=1.44)$ degrees when the concentration of AFP is $10000 \mathrm{ng} / \mathrm{mL}$. The $\Delta \theta$ decreases systematically when the concentration of AFP decreases and $\Delta \theta / \theta_{0}=0.32(\Delta \theta=0.26)$ degrees when the concentration of $\mathrm{AFP}=100 \mathrm{ng} / \mathrm{mL}$. If we further decrease the concentration of AFP to $10 \mathrm{ng} / \mathrm{mL}$, the $\Delta \theta / \theta$ is saturated to $=0.37$. The lowest 


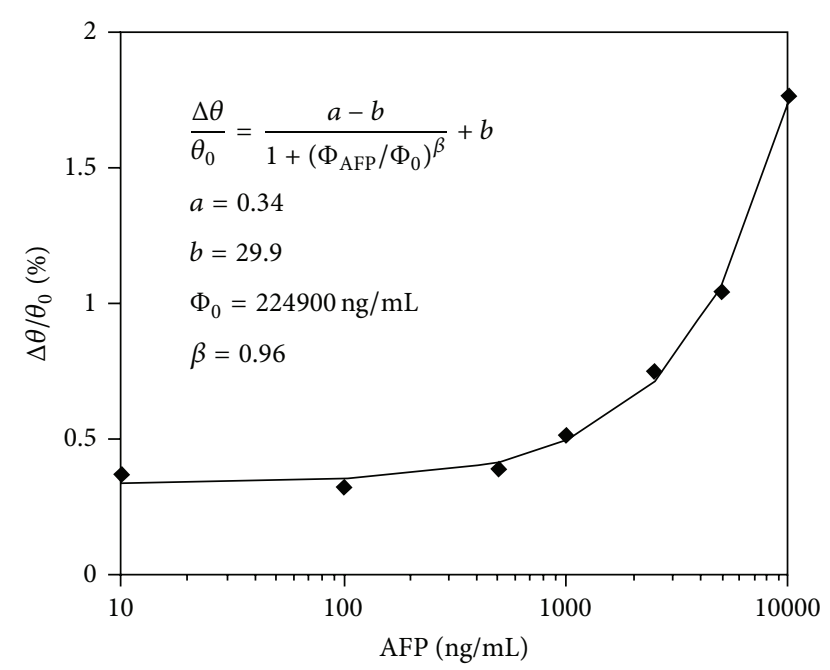

FIgURE 4: $\Delta \theta / \theta_{0}$ as a function of the AFP concentration in a semilog plot.

detection limit is about $100 \mathrm{ng} / \mathrm{mL}$ of AFP in this work. The detection sensitivity can further be improved if we further reduce the noises of the detection system or if SQUID sensors are used [20].

3.2. Dynamic Effective Relaxation Time $\tau_{\text {eff }}$. Figure 5 shows the time dependence of $\tau_{\text {eff }}$ after mixing the reagents with AFP at $300 \mathrm{~K}$. For instance, after mixing the reagent with $5000 \mathrm{ng} / \mathrm{mL}$ AFP, $\tau_{\text {eff }}$ increases from $\tau_{\text {eff }}=0.26 \mu \mathrm{s}$ at $t=0$ to $\tau_{\text {eff }}=0.55 \mu \mathrm{s}$ at $t=6000 \mathrm{~s}$. When assaying $1000 \mathrm{ng} / \mathrm{mL} \mathrm{AFP,}$ we found that $\tau_{\text {eff }}=0.26 \mu \mathrm{s}$ and $\tau_{\text {eff }}=0.38 \mu \mathrm{s}$ at $t=6000 \mathrm{~s}$. When assaying $100 \mathrm{ng} / \mathrm{mL} \mathrm{AFP}$, we found that $\tau_{\text {eff }}=0.26 \mu \mathrm{s}$ at $t=0$ and $\tau_{\text {eff }}=0.32 \mu \mathrm{s}$ at $t=6000 \mathrm{~s}$. It takes $\sim 1.0$ hour to complete the association between $1000 \mathrm{ng} / \mathrm{mL}$ AFP and the reagent. However, the association time is reduced to 0.5 hours when we assay $100 \mathrm{ng} / \mathrm{mL}$ AFP. Consequently, there is a systematic decrease in assaying time when the AFP concentration is reduced. The $\Delta \tau_{\text {eff }}=0.41 \mu \mathrm{s}$ when the concentration of AFP is $10000 \mathrm{ng} / \mathrm{mL}$ and $\Delta \tau_{\text {eff }}=0.06 \mu \mathrm{s}$ when the AFP concentration decreases to $100 \mathrm{ng} / \mathrm{mL}$. If we further reduce the AFP concentration to $10 \mathrm{ng} / \mathrm{mL}$, then $\Delta \tau_{\text {eff }}$ remains as $0.08 \mu \mathrm{s}$. This is the system noise which limits the detection sensitivity. When AFP is conjugated with the reagents which consisted of $\mathrm{Fe}_{3} \mathrm{O}_{4}$-antiAFP, AFP and $\mathrm{Fe}_{3} \mathrm{O}_{4^{-}}$ antiAFP will form magnetic clusters. There is an increase in $\tau_{\text {eff }}$ during the association because of the formation of magnetic clusters, which depresses the Brownian relaxation and therefore increases $\tau_{\text {eff }}$.

Molecule-assisted nanoparticle clustering effect in immunomagnetic reduction assay was reported [21]. In that study, the clustering association was manipulated by controlling the concentrations of BMNs in the reagent. It was found that particle clustering is enhanced by an increase in the concentration of BMNs. As the reagents conjugate with biomarkers, magnetic clusters are formed, which depress the Brownian motion. In this work, we show the phase lag of $\mathbf{M}$ with respect to $\mathbf{H}$, which was characterized to estimate

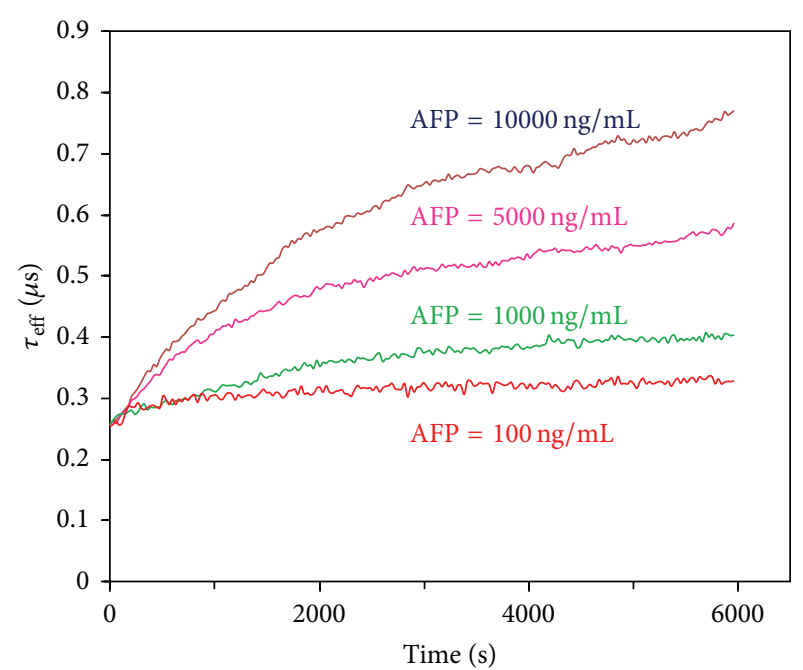

FIGURE 5: Time-dependent $\tau_{\text {eff }}$ for assaying $10000 \mathrm{ng} / \mathrm{mL}, 5000 \mathrm{ng} /$ $\mathrm{mL}, 1000 \mathrm{ng} / \mathrm{mL}$, and $100 \mathrm{ng} / \mathrm{mL}$ of AFP.

the time-dependency of $\tau_{\text {eff }}$ through the following relation: $\tan \theta=\omega \tau_{\text {eff }}$.

A detection scheme for real-time Brownian relaxation of magnetic nanoparticles was also investigated by using a mixed frequency method [22], in which a low frequency of $f_{2}=10 \mathrm{~Hz}$ with a large amplitude and a higher frequency of $20 \mathrm{kHz}$ with a lower amplitude were applied. Instead of detecting the change of amplitudes, the phase delays of the mixed-frequency signals are investigated during the binding process between proteins on BMNs' surface and their respective antibodies. In the present work, instead of using two excitation frequencies, only one exciting frequency was applied with a compensation current to the sensing coil to achieve a high balance. Additionally, the real-time $\theta$ is measured to characterize real-time $\tau_{\text {eff }}$. By analyzing the changes of real-time $\tau_{\text {eff }}$, a detection sensitivity of human AFP better than $100 \mathrm{ng} / \mathrm{mL}$ is demonstrated.

3.3. Universal Logistic Function in $\Delta \tau_{\text {eff }} / \tau_{0}$. Figure 6 shows the normalized effective relaxation time reduction $\Delta \tau_{\text {eff }} / \tau_{0}$ as a function of AFP concentration in a semilog plot at $300 \mathrm{~K}$, where $\tau_{0}=0.26 \mu \mathrm{s}$ is the effective relaxation time at $t=0$. The $\Delta \tau_{\text {eff }} / \tau_{0}=1.9$ for assaying $10000 \mathrm{ng} / \mathrm{mL}$ of AFP, and then the $\Delta \tau_{\text {eff }} / \tau_{0}$ decreases to 0.61 when we assay $1000 \mathrm{ng} / \mathrm{mL}$ of AFP and the $\Delta \tau_{\text {eff }} / \tau_{0}$ is further saturated to 0.35 when we assay $10 \mathrm{ng} / \mathrm{mL}$.

The $\Delta \tau_{\text {eff }} / \tau_{0}$ as a function of AFP concentration will follow a universal characteristic logistic function as follows [16]:

$$
\frac{\Delta \tau_{\mathrm{eff}}}{\tau_{0}}=\frac{(a-b)}{\left\{1+\left[\left(\Phi_{\mathrm{AFP}}\right) /\left(\Phi_{0}\right)\right]^{\beta}\right\}}+b,
$$

where $\tau_{0}$ is the effective relaxation time at $t=0 . a, b$, and $\gamma$ are dimensionless parameters. $\Phi_{\mathrm{AFP}}$ and $\Phi_{0}$ are in units of $\mathrm{ng} / \mathrm{mL}$. The solid line is the fitting curve with parameters $a=0.34, b=29.9, \Phi_{0}=224900 \mathrm{ng} / \mathrm{mL}$, and $\beta=0.961$. Due 


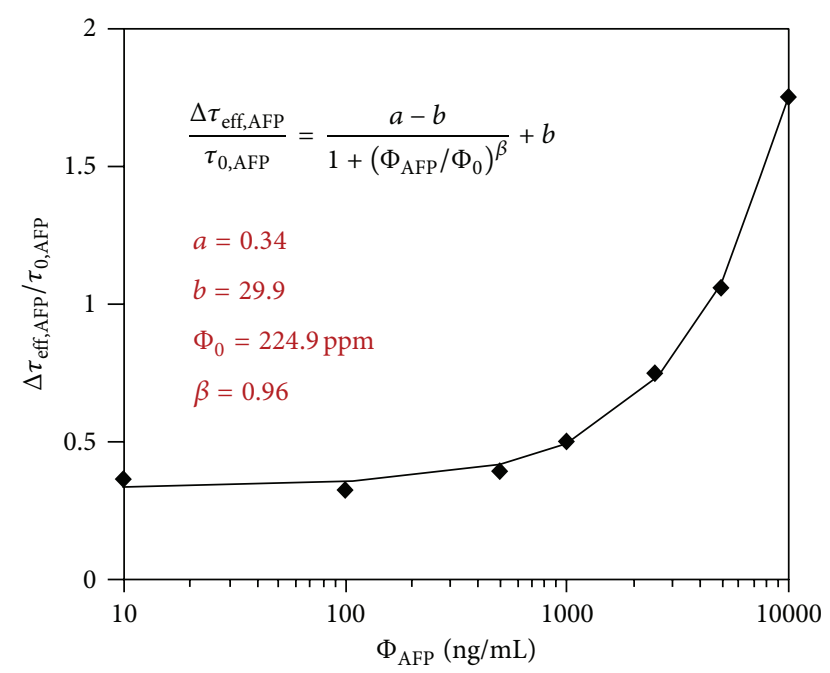

Figure 6: $\Delta \tau_{\text {eff }} / \tau_{0}$ as a function of the AFP concentration in a semilog plot. The solid line is the fitting curve to (4).

to the presence of noises, the $\Delta \tau_{\text {eff }} / \tau_{0}$ signal shows a nonzero value of $\Phi_{\mathrm{AFP}}=0$. This universal logistic function provides a basis for estimating the unknown amount of biomolecules [16]. Versatile categories of bioentities, for example, proteins, viruses, small-molecule chemicals, and cytokines, despite being different from each other, have all shown to behave similarly, resulting in a general logistic function for all biotargets. The present concentration-dependent $\Delta \tau_{\text {eff }} / \tau_{0}$ also shows this behavior of logistic function, which provides a foundation for assaying an unknown amount of biomolecules. The detection threshold of the detected signal is defined as that higher than the noise level of the detected signal at low concentrations. In this work, the lowest noise level of $\Delta \tau_{\text {eff }} / \tau_{0}$ is about 0.35 . Therefore, the detection threshold of AFP concentration can be determined via (4), which results in $55 \mathrm{ng} / \mathrm{mL}$.

Figure 7(a) shows the magnetization as a function of the applied magnetic field for different concentrations of AFP when the association of $\mathrm{Fe}_{3} \mathrm{O}_{4}$-antiAFP with AFP is complete. The magnetization was measured with a vibrating sample magnetometer. The concentrations of AFP varied from $10 \mathrm{ng} / \mathrm{mL}$ to $10000 \mathrm{ng} / \mathrm{mL}$. It was found that the $M_{s}$ increases when the concentration of AFP increases. The increased $M_{s}$ is due to the fact that more magnetic clusters of $\mathrm{Fe}_{3} \mathrm{O}_{4}$-antiAFP-AFP are formed during the association. The $\Delta M_{s}$ is $0.32 \mathrm{emu} / \mathrm{g}$ when the concentration of AFP is $10000 \mathrm{ng} / \mathrm{mL}$ and $\Delta M_{s}$ decreases to $0.06 \mathrm{emu} / \mathrm{g}$ when the concentration is $10 \mathrm{ng} / \mathrm{mL}$, where $\Delta M_{s}$ is the change $M_{s}$ at $H=1 \mathrm{~T}$ in the reagent with and without AFP. The $\Delta M_{s, \mathrm{AFP}} / M_{s, 0}$ as a function of AFP concentration is shown in Figure 7(b). As the concentration of AFP decreases, less magnetic clusters are formed. Therefore the $\Delta M_{s}$ decreases monotonically when the concentration of AFP decreases. The $\Delta M_{s}$ reaches a saturated behavior when the concentration of $\mathrm{AFP}$ is $10 \mathrm{ng} / \mathrm{mL}$. Hence, a detection sensitivity of $10 \mathrm{ng} / \mathrm{mL}$ of AFP by measuring the variation of saturated magnetization is demonstrated.
The $\Delta M_{s, \mathrm{AFP}} / M_{s, 0}$ data are fitted to the universal logistic function:

$$
\frac{\Delta M_{s, \mathrm{AFP}}}{M_{s, 0}}=\frac{(A-B)}{\left\{1+\left[\left(\Phi_{\mathrm{AFP}}\right) /\left(\Phi_{0}\right)\right]^{\beta}\right\}}+B,
$$

where $M_{s, 0}$ is the saturated magnetization of $\mathrm{Fe}_{3} \mathrm{O}_{4}$-antiAFP. The fitting parameters $A$ and $B$ are in units of emu/g, while $\Phi_{0}$ is in units of $\mathrm{ng} / \mathrm{mL}$, and $\beta$ is dimensionless. The solid curve is the fitted line with parameters $A=0.62 \mathrm{emu} / \mathrm{g}$, $B=4.3 \mathrm{emu} / \mathrm{g}, \Phi_{0}=5430 \mathrm{ng} / \mathrm{mL}$, and $\beta=0.88$. This solid curve provides a foundation for assaying unknown amounts of AFP.

The molecule-assisted nanoparticle clustering effect was reported in immunomagnetic reduction assay [21]. In that study, magnetic particle clustering was manipulated by controlling the concentrations of antibody-functionalized magnetic nanoparticles in the reagent. The results show that particle clustering is enhanced by an increase in the concentration of the to-be-detected biotargets. In the present study, we found that $M_{s}$ increases when the concentration of AFP increases. This is due to the fact that more magnetic clusters are associated in the reagent, and therefore the $M_{s}$ increases when the concentration of AFP increases.

Figure 8 shows the representative time-dependent amplitude, $\chi_{\text {amp }}$, for assaying $10000 \mathrm{ng} / \mathrm{mL}$ AFP (Figure 8(a)) and $50 \mathrm{ng} / \mathrm{mL}$ AFP (Figure 8(b)). For assaying $10000 \mathrm{ng} / \mathrm{mL}$ AFP, $\chi_{\mathrm{amp}}=13.4 \mu \mathrm{V}$ at $t=0$ and decreases to $12.06 \mu \mathrm{V}$ at $t=6000 \mathrm{~s}$. Therefore, we obtain $\Delta \chi_{\mathrm{amp}} / \chi_{\mathrm{amp}}(t=0)=10 \%$ for assaying $10000 \mathrm{ng} / \mathrm{mL}$ AFP. For assaying $100 \mathrm{ng} / \mathrm{mL}$ AFP, $\chi_{\mathrm{amp}}=12.33 \mu \mathrm{V}$ at $t=0$ and decreases to $12.22 \mu \mathrm{V}$ at $t=6000 \mathrm{~s}$. Therefore, $\Delta \chi_{\mathrm{amp}} / \chi_{\mathrm{amp}}(t=0)=0.9 \%$ for assaying $100 \mathrm{ng} / \mathrm{mL}$ AFP. It was observed that the amplitude reduction decreases when the concentration of AFP decreases. The amplitude of $(1)$ is $\chi_{\mathrm{amp}}=\chi_{0}\left\{\left\{1 /\left[1+\left(\omega \tau_{\text {eff }}\right)^{2}\right]\right\}^{2}+\left\{\left(\omega \tau_{\text {eff }}\right) /[1+\right.\right.$ $\left.\left.\left.\left(\omega \tau_{\text {eff }}\right)^{2}\right]\right\}^{2}\right\}^{1 / 2}=\chi_{0}\left\{1 /\left[1+\left(\omega \tau_{\text {eff }}\right)^{2}\right]^{1 / 2}\right\}$. Therefore an increase in $\tau_{\text {eff }}$ during the association will cause a reduction in $\chi_{\text {amp. }}$. We have shown that the magnetic clustering effect is enhanced by increasing the concentration of the AFP. Furthermore, the amplitude reduction shows characteristics similar to that observed in a mixed-frequency IMR [21].

A method of wash-free IMR assay using mixed excitation frequencies was previously proposed [9]. In such detection, the sensing coils consist of two excitation coils and one pickup coil. Two excitation currents at frequencies $f_{1}(t)$ and $f_{2}(t)$ were applied to the excitation coils. The reduction of $\chi_{\mathrm{ac}}$ for the BMNs conjugated with the target biomarkers is analyzed at a target frequency of $f_{1}(t)+2 f_{2}(t)$ to qualitatively determine the amount of biomarkers, where $f_{1}(t)$ and $f_{2}(t)$ are the excitation frequencies of the input coils. This mixedfrequency ac susceptibility reduction has been successfully used to assay AFP [17], which shows a high sensitivity and specificity. In the present work a detection threshold better than $100 \mathrm{ng} / \mathrm{mL}$ is demonstrated by characterizing the change in the effective relaxation time. The present biosystem has been applied to detect the $\mathrm{C}$-reactive protein via the characterization of $\Delta \theta_{\text {eff }} / \theta_{0}$ [18]. In practice, the reference criteria of the AFP serum level for the diagnosis of hepatocellular carcinoma (HCC) are above $20 \mathrm{ng} / \mathrm{mL}$. To achieve a higher 


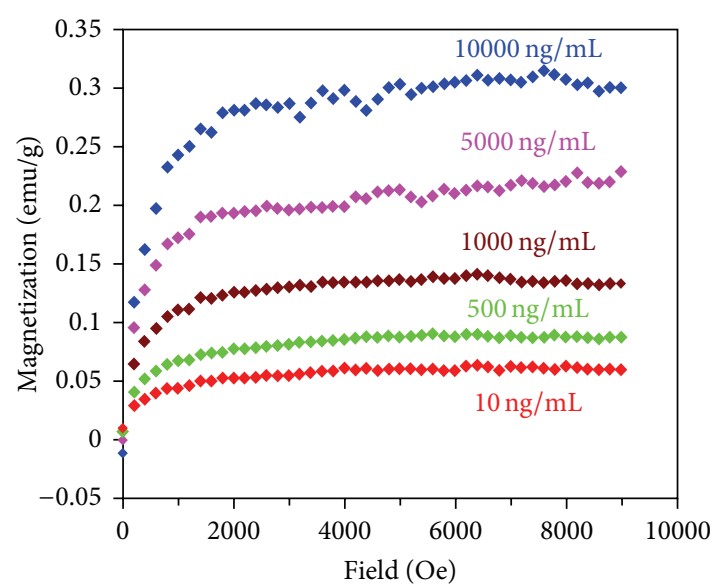

(a)

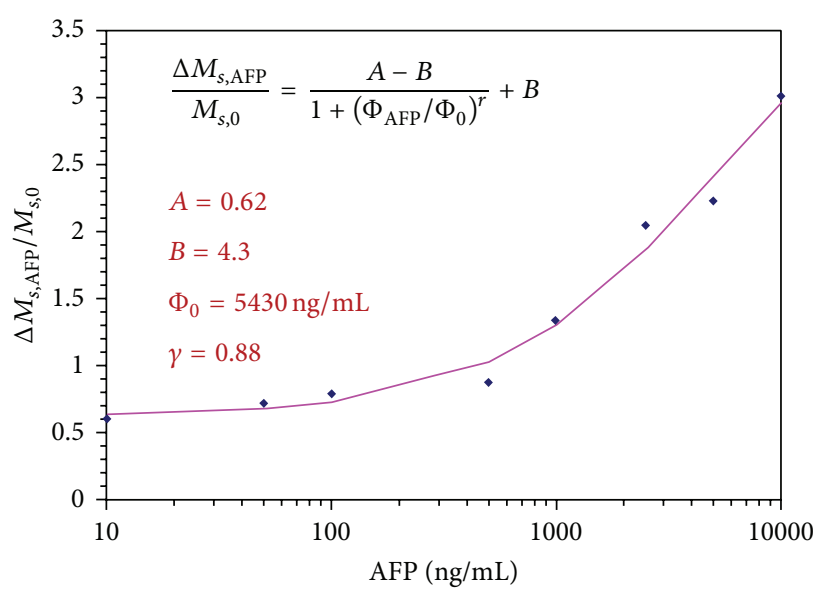

(b)

FIGURE 7: (a) Magnetization as a function of applied magnetic field for different concentrations of AFP when the association of Fe $\mathrm{O}_{4}$-antiAFP with AFP is complete and (b) $\Delta M_{s}$ as a function of AFP concentration.

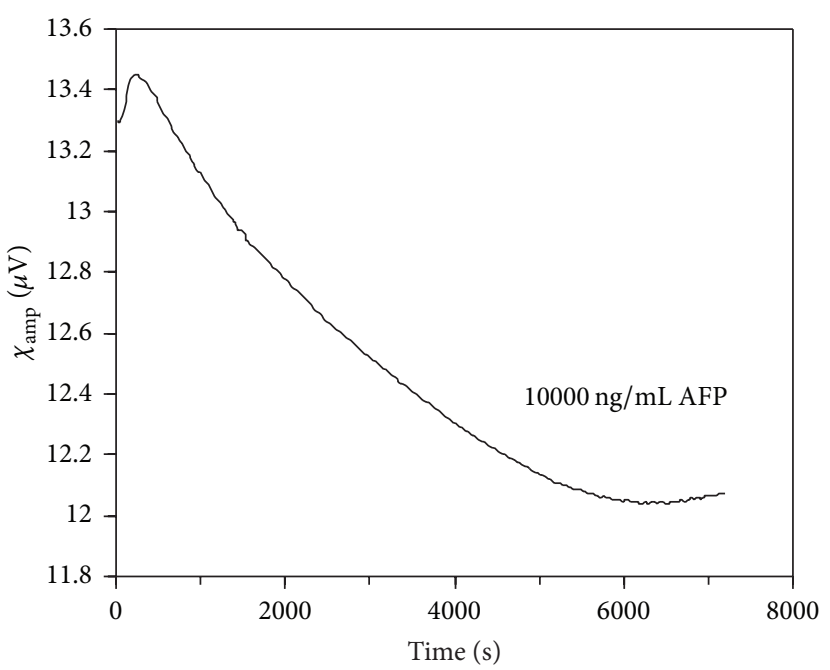

(a)

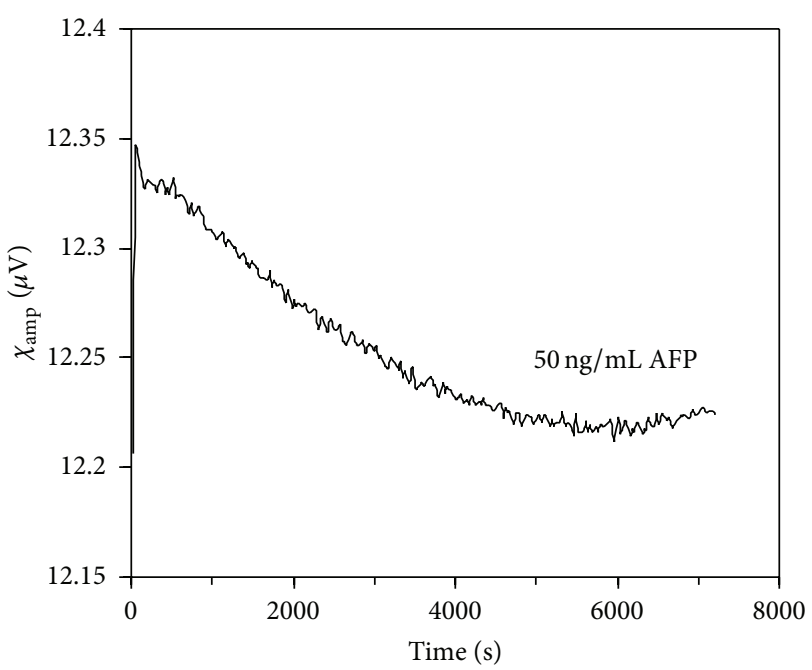

(b)

FIGURE 8: Representative time-dependent ac susceptibility for assaying (a) $10000 \mathrm{ng} / \mathrm{mL}$ AFP and (b) $50 \mathrm{ng} / \mathrm{mL}$ AFP.

detection sensitivity, we can couple the sensing coil to high$T_{c}$ SQUID via a flux transformer [20].

\section{Conclusion}

In this work, we report a platform for assaying biomarkers of AFP by using reagents that consisted of $\mathrm{Fe}_{3} \mathrm{O}_{4}$-antiAFP and a highly sensitive ac susceptometer. By monitoring $\theta$, we found that the effective relaxation time increases monotonically and becomes saturated when the association between AFP and $\mathrm{Fe}_{3} \mathrm{O}_{4}$-antiAFP is complete. Additionally, the change of $\tau_{\text {eff }}$ and $M_{s}$ increases when the concentration of AFP increases after the association. This is due to the fact that more and larger magnetic clusters consisting of $\mathrm{Fe}_{3} \mathrm{O}_{4}$-antiAFPAFP are formed during the association. The concentrationdependent $\Delta \tau_{\text {eff }} / \tau_{0}$ and $\Delta M_{s, \mathrm{AFP}} / M_{s, 0}$ show a universal behavior of the logistic function, which provides a foundation for estimating an unknown amount of biomolecules. The detection platforms are robust and easy to use and show promise for further use in biomedical applications.

\section{Conflict of Interests}

The authors declare that there is no conflict of interests regarding the publication of this paper.

\section{Acknowledgments}

This work is supported under Grants nos. NSC101-2120M-168-001, NSC99-2112-M-168-001-MY3, NSC102-2112M-003-017, NSC101-2112-M-003-009, NSC102-2923-M003- 
001, NSC102-2622-M002-001-CC2, NSC102-2112-M-003008-MY2, and NSC 100-2112-M-003-011-MY2 and the Ministry of Health and Welfare under Grants nos. DOH102TD-PB-111-TM007 and DOH102-TD-N-111-002.

\section{References}

[1] G. Zheng, F. Patolsky, Y. Cui, W. U. Wang, and C. M. Lieber, "Multiplexed electrical detection of cancer markers with nanowire sensor arrays," Nature Biotechnology, vol. 23, no. 10, pp. 1294-1301, 2005.

[2] K. Aslan, J. R. Lakowicz, and C. D. Geddes, "Plasmon light scattering in biology and medicine: New sensing approaches, visions and perspectives," Current Opinion in Chemical Biology, vol. 9, no. 5, pp. 538-544, 2005.

[3] S. Y. Yang, R. M. Wu, Z. F. Jian, H. E. Horng, C.-Y. Hong, and H. C. Yang, "One-sample measurement in laser nephelometric immunoassay using magnetic nanoparticles," Applied Physics Letters, vol. 89, no. 24, Article ID 244102, 2006.

[4] C. R. Tamanaha, S. P. Mulvaney, J. C. Rife, and L. J. Whitman, "Magnetic labeling, detection, and system integration," Biosensors and Bioelectronics, vol. 24, no. 1, pp. 1-13, 2008.

[5] D. R. Baselt, G. U. Lee, M. Natesan, S. W. Metzger, P. E. Sheehan, and R. J. Colton, "A biosensor based on magnetoresistance technology," Biosensors and Bioelectronics, vol. 13, no. 7-8, pp. 731-739, 1998.

[6] R. Kötitz, W. Weitschies, L. Trahms, W. Brewer, and W. Semmler, "Determination of the binding reaction between avidin and biotin by relaxation measurements of magnetic nanoparticles," Journal of Magnetism and Magnetic Materials, vol. 194, no. 1, pp. 62-68, 1999.

[7] S. K. Lee, W. R. Myers, H. L. Grossman, H.-M. Cho, Y. R. Chemla, and J. Clarke, "Magnetic gradiometer based on a hightransition temperature superconducting quantum interference device for improved sensitivity of a biosensor," Applied Physics Letters, vol. 81, no. 16, pp. 3094-3096, 2002.

[8] K. Enpuku, T. Minotani, T. Gima et al., "Detection of magnetic nanoparticles with superconducting quantum interference device (SQUID) magnetometer and application to immunoassays," Japanese Journal of Applied Physics, vol. 38, pp. L1102L1105, 1999.

[9] C.-Y. Hong, C. C. Wu, Y. C. Chiu, S. Y. Yang, H. E. Horng, and H. C. Yang, "Magnetic susceptibility reduction method for magnetically labeled immunoassay," Applied Physics Letters, vol. 88, no. 21, Article ID 212512, 2006.

[10] H. E. Horng, S. Y. Yang, C.-Y. Hong et al., "Biofunctionalized magnetic nanoparticles for high-sensitivity immunomagnetic detection of human C-reactive protein," Applied Physics Letters, vol. 88, no. 25, Article ID 252506, 2006.

[11] H. Lee, E. Sun, D. Ham, and R. Weissleder, "Chip-NMR biosensor for detection and molecular analysis of cells," Nature Medicine, vol. 14, no. 8, pp. 869-874, 2008.

[12] Y. R. Chemla, H. L. Grossman, Y. Poon et al., "Ultrasensitive magnetic biosensor for homogeneous immunoassay," Proceedings of the National Academy of Sciences of the United States of America, vol. 97, no. 26, pp. 14268-14272, 2000.

[13] D. R. Baselt, G. U. Lee, M. Natesan, S. W. Metzger, P. E. Sheehan, and R. J. Colton, "A biosensor based on magnetoresistance technology," Biosensors and Bioelectronics, vol. 13, no. 7-8, pp. 731-739, 1998.
[14] T. Aytur, J. Foley, M. Anwar, B. Boser, E. Harris, and P. R. Beatty, "A novel magnetic bead bioassay platform using a microchip-based sensor for infectious disease diagnosis," Journal of Immunological Methods, vol. 314, no. 1-2, pp. 21-29, 2006.

[15] H. Shao, C. Min, D. Issadore et al., "Magnetic nanoparticles and micronmr for diagnostic applications," Theranostics, vol. 2, no. 1, pp. 55-65, 2012.

[16] C. C. Yang, S. Y. Yang, J. J. Chieh, H. E. Horng, C. Y. Hong, and H. C. Yang, "Universal behavior of biomolecule-concentrationdependent reduction in AC magnetic susceptibility of bioreagents," IEEE Magnetics Letters, vol. 3, p. 1500104, 2012.

[17] K.-W. Huang, S.-Y. Yang, Y.-W. Hong et al., "Feasibility studies for assaying alpha-fetoprotein using antibody-activated magnetic nanoparticles," International Journal of Nanomedicine, vol. 7, pp. 1991-1996, 2012.

[18] S. H. Liao, H. C. Yang, H. E. Horng et al., "Time-dependent phase lag of biofunctionalized magnetic nanoparticles conjugated with biotargets studied with alternating current magnetic susceptometor for liquid phase immunoassays," Applied Physics Letters, vol. 103, no. 24, Article ID 243703, 2013.

[19] R. E. Rosensweig, "Heating magnetic fluid with alternating magnetic field," Journal of Magnetism and Magnetic Materials, vol. 252, pp. 370-374, 2002.

[20] S. Y. Yang, J. J. Chieh, W. C. Wang et al., "Magnetic nanoparticles for high-sensitivity detection on nucleic acids via superconducting-quantum-interference-device-based immunomagnetic reduction assay," Journal of Magnetism and Magnetic Materials, vol. 323, no. 6, pp. 681-685, 2011.

[21] S. Y. Yang, J. J. Chieh, K. W. Huang et al., "Molecule-assisted nanoparticle clustering effect in immunomagnetic reduction assay," Journal of Applied Physics, vol. 113, no. 14, Article ID 144903, 2013.

[22] L. Tu, Y. Jing, Y. Li, and J.-P. Wang, "Real-time measurement of Brownian relaxation of magnetic nanoparticles by a mixingfrequency method," Applied Physics Letters, vol. 98, no. 21, Article ID 213702, 2011. 

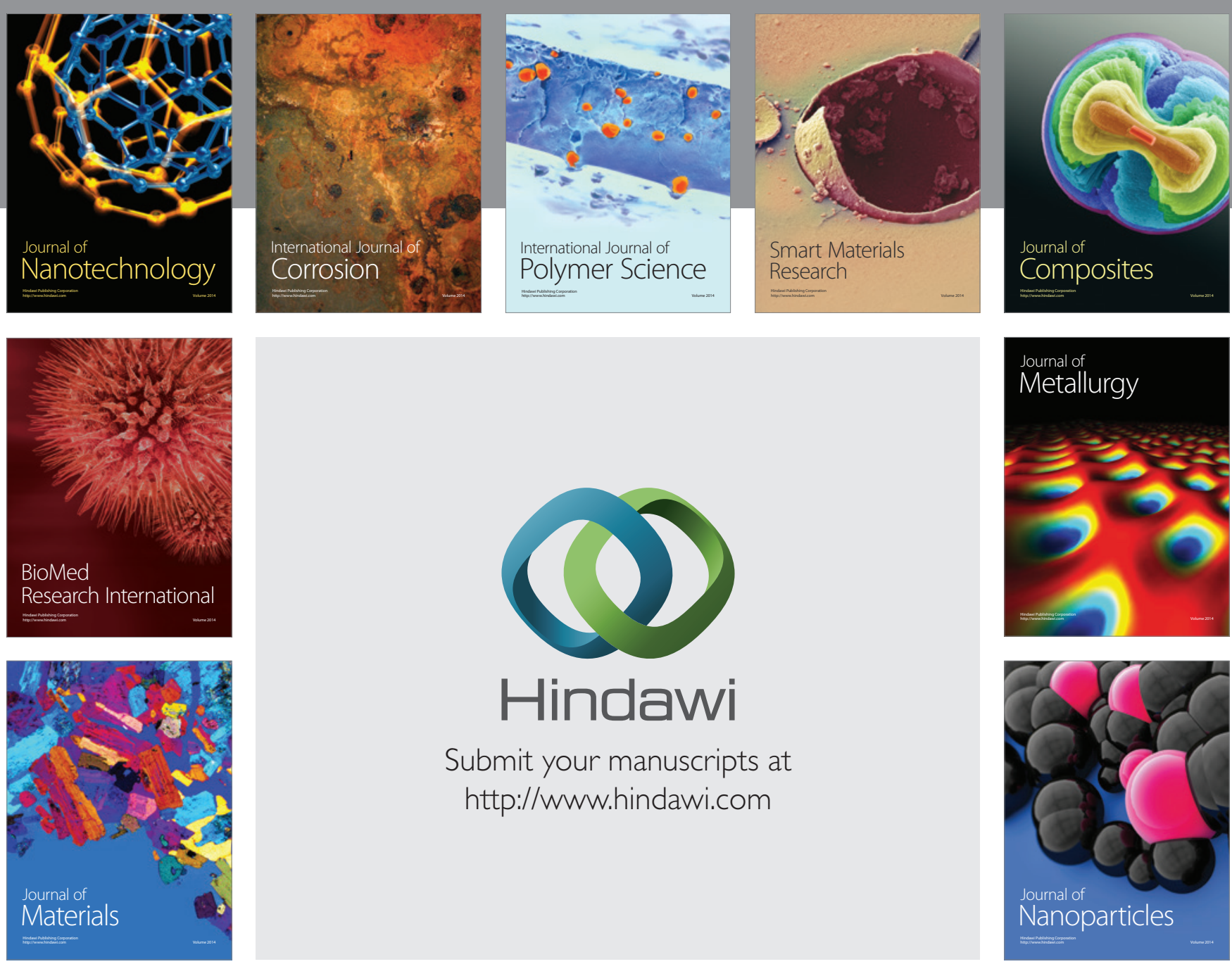

Submit your manuscripts at http://www.hindawi.com
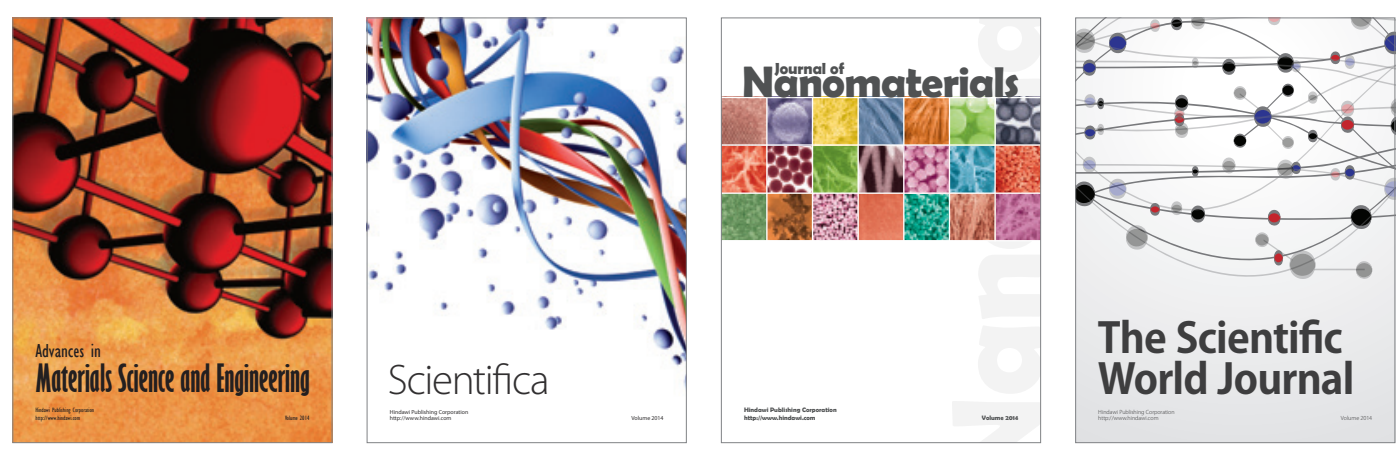

\section{The Scientific World Journal}
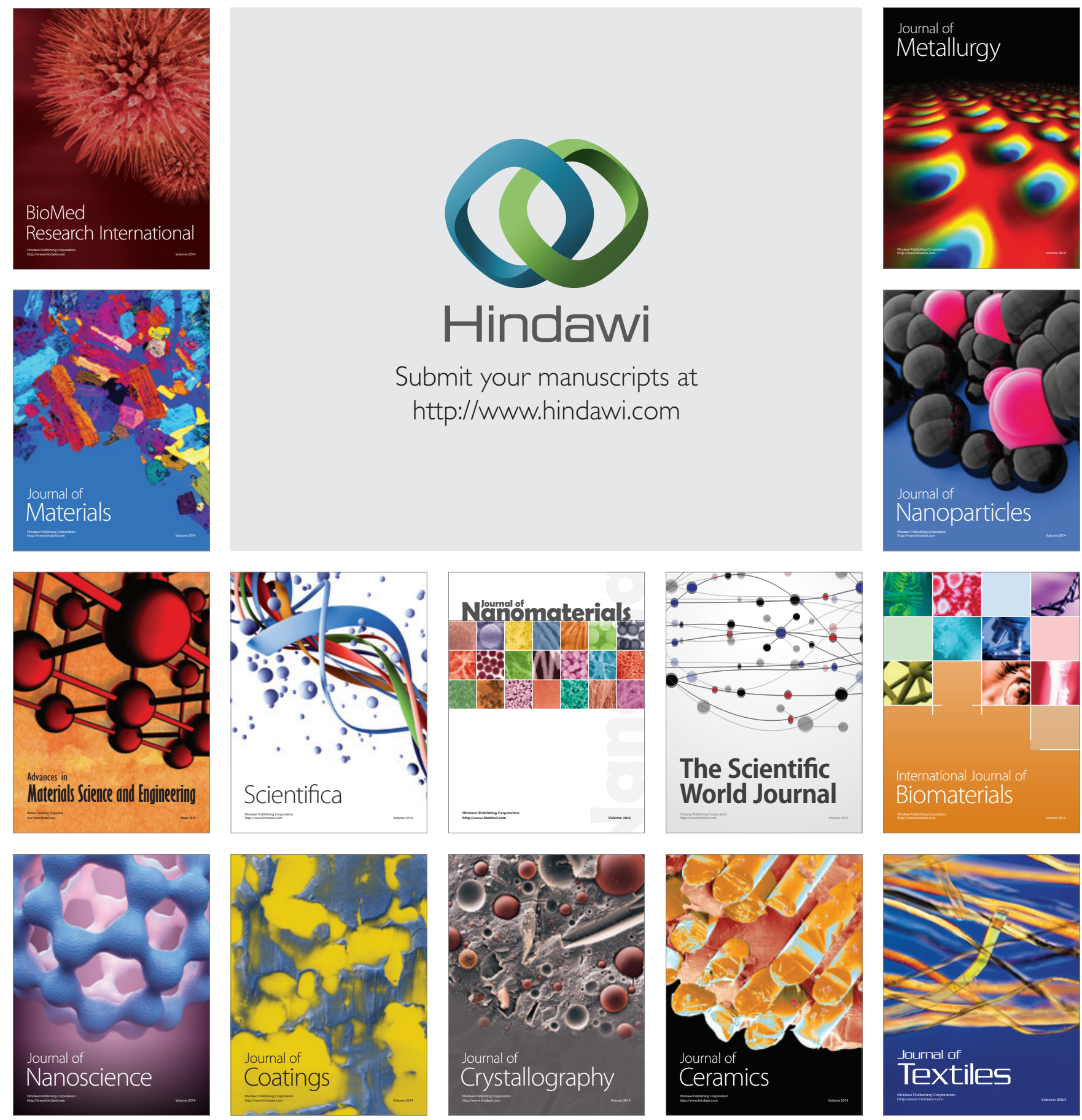